\title{
Andrographolide enhanced 5-fluorouracil-induced antitumor effect in colorectal cancer via inhibition of c-MET pathway
}

This article was published in the following Dove Press journal:

Drug Design, Development and Therapy

Number of times this article has been viewed

\author{
Meng Su' \\ Baoli Qin' \\ Fang Liu \\ Yuze Chen ${ }^{2}$ \\ Rui Zhang ${ }^{2}$ \\ 'Department of Internal Medicine, \\ ${ }^{2}$ Department of Colorectal Surgery, \\ Cancer Hospital of China Medical \\ University, Liaoning Cancer Hospital \\ and Institute, Liaoning, China
}

Correspondence: Rui Zhang Department of Colorectal Surgery, Cancer Hospital of China Medical University, Liaoning Cancer Hospital and Institute, 44 Xiaoheyan Road, Dadong District, Shenyang I 10042, Liaoning

Province, China

$\mathrm{Tel}+8602431916293$

Email rui_zhangII@I26.com

\begin{abstract}
Colorectal cancer (CRC) is the third most common malignant neoplasm worldwide. 5-Fluorouracil $(5-\mathrm{Fu})$ is the most important chemotherapeutic drug used for the treatment of $\mathrm{CRC}$. However, resistance to 5-Fu therapies is a growing concern in $\mathrm{CRC}$ clinical practice recently. Andrographolide (Andro) is a main bioactive constituent of the herb Andrographis paniculata, which has various biological effects including anti-inflammation and antitumor activities. In the present study, we investigated the effects of combined Andro with 5-Fu against CRC HCT-116 cells. In vitro studies showed that Andro synergistically enhanced the anti-proliferation effect of 5-Fu on HCT-116 cells due to increased apoptotic cells. Meanwhile, results of the enzyme linked immunosorbent assay indicated that the level of phosphorylated cellular-mesenchymal to epithelial transition factor ( $\mathrm{p}-\mathrm{MET}$ ) was decreased by the combination treatment. Further study suggested that Andro promoted the antitumor effect of 5-Fu by downregulating the level of p-MET. In conclusion, these results confirmed the synergistic antitumor activity of Andro on CRC and provide evidence for possible clinical application of Andro for enhancing the antitumor effect of 5-Fu in CRC treatment.
\end{abstract}

Keywords: Andro, 5-Fu, HCT-116 cells, apoptosis, p-MET

\section{Introduction}

Colorectal cancer (CRC) is the third most common malignant neoplasm worldwide and causes $\sim 600,000$ deaths annually, which makes it the fourth common cause of cancer-related death. ${ }^{1,2}$ Despite advances in modern medical technology, CRC patients still face high mortality and poor prognosis due to local relapse, metastasis, and resistance to chemotherapy., ${ }^{3,4}$ The mainstay of chemotherapy treatment of CRC is 5-fluorouracil (5-Fu), which exerts its therapeutic effect via DNA damage-triggered signaling. ${ }^{5}$ Although 5-Fu provides effective chemotherapy treatment, resistance to 5 -Fu is one of the major reasons for poor prognosis of patients during cancer treatment. ${ }^{6}$ It is therefore necessary to find a potential agent which could enhance the sensibility of 5-Fu and reduce its dosage.

Andrographolide (Andro) is a main bioactive constituent of the herb Andrographis paniculata, which is usually used in Chinese medicine to treat diverse diseases. ${ }^{7}$ Recent studies showed that Andro was an effective agent with its pharmacological bioactive properties, including anti-inflammation, antibacterial, anti-tuberculosis, antitumor properties, and so on. ${ }^{8-11}$ Other studies have also demonstrated the antitumor mechanisms of Andro. For example, Andro could resensitize cisplatin resistance against non-small cell lung carcinoma via activation of the Akt/mTOR pathway. ${ }^{12}$ In addition, 
Andro induced apoptosis via endoplasmic reticulum stress and IRE-1 signaling against colon cancer. ${ }^{13}$ Furthermore, one study found that Andro reversed 5-Fu resistance in human CRC via elevating Bax expression. ${ }^{14}$ Thus, we suggest that Andro could be a potential agent to enhance the antitumor effect of 5-Fu against CRC.

In the current study, we investigated the effect of combination treatments of 5-Fu with Andro against CRC HCT-116 in vitro and in vivo and explored the underlying mechanisms of the treatments. Our results elucidated that 5-Fu plus Andro inhibited cell growth via inducing apoptosis, and the bioactive property was attributed to regulating the expression level of phosphorylated cellular-mesenchymal to epithelial transition factor ( $\mathrm{p}-\mathrm{MET}$ ) in vivo and in vitro. These results provide evidence for the possible clinical application of Andro for enhancing the effect of 5-Fu on CRC.

\section{Methods and materials Reagents}

Andro, with a purity $>98 \%$, was obtained from National Institutes for Food and Drug Control, China. 5-Fu was purchased from Sigma-Aldrich Co (St Louis, MO, USA). Dimethyl sulfoxide (DMSO), trypsin, phosphate-buffered saline (PBS), 3-(4,5-dimethyl-2-thiazolyl)-2,5-diphenyl2H-tetrazolium bromide (MTT), propidium iodide (PI), Annexin V, and hepatocyte growth factor (HGF) were purchased from Thermo Fisher Scientific (Waltham, MA, USA). Primary antibodies against p-AKT, p-ERK, c-MET, p-MET, and GAPDH were purchased from Santa Cruz Biotechnology Inc (Dallas, TX, USA) with 1:250 dilution. All chemicals were directly used without further purification.

\section{Cell lines}

Human colorectal carcinoma HCT-116 cells were obtained from the American Type Culture Collection (ATCC; Manassas, VA, USA) (HCT-116 [ATCC ${ }^{\circledR}$ CCL-247TM]), and cultured in minimum essential medium supplemented with $100 \mu \mathrm{g} / \mathrm{mL}$ streptomycin, $100 \mathrm{U} / \mathrm{mL}$ penicillin, and $10 \%$ fetal bovine serum at $37^{\circ} \mathrm{C}$ with $5 \% \mathrm{CO}_{2}$ (Sigma Aldrich Co.). Cells were routinely passaged every 3 days. They were treated for indicated hours with medium change and fresh drug.

\section{Cell growth inhibition assay}

Cell growth inhibition was measured by the MTT assay. Briefly, HCT-116 cells were seeded in triplicate into 96-well plates (Corning, NY, USA) with a density of $1 \times 10^{4}$ cells/well. After incubating overnight, the cell medium was removed, and the cells were then subjected to different concentrations of 5-Fu, or the combination of 5-Fu with Andro. They were then incubated for 24,48 , and 72 hours. The medium was removed, and MTT was immediately added into the wells at a final concentration of $0.5 \mathrm{mg} / \mathrm{mL}$ at $37^{\circ} \mathrm{C}$ and incubated for 3 hours; the medium was completely removed and formazan crystals were resolved in DMSO, and the absorbance measured at $492 \mathrm{~nm}$ using a plate reader (Thermo Fisher Scientific). Cell growth inhibition was calculated by using the following formula:

Cell growth inhibition $(\%)=$

$$
100 \times\left(1-\frac{\text { OD value of experimental sample group }}{\text { OD value of control group }}\right)
$$

$\mathrm{IC}_{50}$ value was calculated using GraphPad Prism software V5.0 for Windows (GraphPad Software Inc, La Jolla, CA, USA).

\section{Observation of cell morphologic changes}

HCT-116 cells were seeded into 6-well plates at a density of $5 \times 10^{4} / \mathrm{mL}$. The cells were cultured at $37^{\circ} \mathrm{C}, 5 \% \mathrm{CO}_{2}$ overnight. Later, the culture medium was removed and the cells were treated with indicated treatments. DMSO $(0.1 \%)$ was used as the control group. The cell morphologic changes were observed and captured by an optical microscope (Nikon Eclipse Ti-S; Nikon Corporation, Tokyo, Japan) after 24 hours culturing.

\section{Analysis of drug synergism}

The combination profile of Andro and 5-Fu was analyzed using combination index (CI) according to Chou's method. ${ }^{15-17}$ Data from the cell viability assay were employed to perform this statistical analysis. The CI value was calculated as follows:

$$
\mathrm{CI}=\frac{\mathrm{C}_{\mathrm{A}, \mathrm{x}}}{\mathrm{IC}_{50, \mathrm{~A}}}+\frac{\mathrm{C}_{\mathrm{B}, \mathrm{x}}}{\mathrm{IC}_{50, \mathrm{~B}}}
$$

where $\mathrm{C}_{\mathrm{A}, \mathrm{x}}$ and $\mathrm{C}_{\mathrm{B}, \mathrm{x}}$ represent the concentration of a single agent to achieve the antitumor effect $\mathrm{x}$ of combination $\operatorname{drug} \mathrm{A}$ with $\mathrm{B}$, and $\mathrm{IC}_{50, \mathrm{X}}$ is the $\mathrm{IC}_{50}$ value of drug $\mathrm{X}$. CI values $<1$ indicate a synergistic profile,$=1$ indicate an additive profile, and $>1$ indicate an antagonistic profile of the association tested, according to Chou. ${ }^{16}$

\section{Western blot analysis}

HCT-116 cells were seeded into 6-well plates at a density of $1 \times 10^{5}$ cells/well, and incubated overnight at $37^{\circ} \mathrm{C}$ with $5 \% \mathrm{CO}_{2}$. Then, the cells were subjected to the indicated 
treatments $(0.1 \%$ DMSO, $4.28 \mu \mathrm{M} 5-\mathrm{Fu}, 10 \mu \mathrm{M}$ Andro, and combination treatment of 5-Fu with Andro). The cells were collected and lysed using real-time instant presence with advertisement in 72 hours. The cell concentration of each group was measured by BCA Protein Assay Kit (Pierce Biotechnology, Rockford, IL, USA). The proteins mixed with loading buffer were boiled for 5 minutes. Then, they were subjected to electrophoresis with same protein loading on $10 \%$ sodium dodecyl sulfate-polyacrylamide gel electrophoresis gel for 2 hours for separation, and transferred to Millipore Immobilon-P Transfer Membranes (EMD Millipore, Billerica, MA, USA) at $100 \mathrm{~mA}$ for 2 hours. The membranes were blocked in 5\% milk at room temperature, and then incubated with primary antibodies at $4^{\circ} \mathrm{C}$ overnight. Later, the membranes were washed with cold PBS three times and then incubated with the corresponding secondary antibodies $(1: 1,000)$ at room temperature for 2 hours. The expression levels of proteins were detected by electrochemiluminescence (Thermo Fisher Scientific).

\section{Cell apoptosis assay}

Annexin V demonstrates high affinity to phospholipid phosphatidylserine, which could transpose to the external side of the plasma membrane during early apoptosis. ${ }^{18} \mathrm{PI}$ was used to investigate changes in cell nuclear morphology, apoptosis, and necrosis. ${ }^{19}$ Cell apoptosis assay was observed using PI/Annexin V double staining and flow cytometry. HCT-116 cells were seeded into 6-well plates at a density of $2 \times 10^{5} /$ well overnight. Then, they were subjected to the indicated treatments $(0.1 \%$ DMSO, $4.28 \mu \mathrm{M} 5-\mathrm{Fu}, 10 \mu \mathrm{M}$ Andro, and combination treatment of 5-Fu with Andro). After 72 hours incubation, the cells were trypsinized, and washed twice with cold PBS buffer. Then, the cells were centrifuged and resupended in Annexin V and PI reagent buffer according to the manufacturer's manual. Subsequently, the apoptosis rate was measured by flow cytometry (FACSCalibur; BD Biosciences, Franklin Lakes, NJ, USA).

\section{Enzyme-linked immunosorbent assay (ELISA)}

The expression levels of potential proteins were measured using ELISA kits (R\&D Systems, Minneapolis, MN, USA) according to the manufacturer's manuals. In brief, the cells were subjected to the indicated treatments $(0.1 \% \mathrm{DMSO}$, $4.28 \mu \mathrm{M} 5-\mathrm{Fu}, 10 \mu \mathrm{M}$ Andro, and combination treatment of 5-Fu with Andro) for 72 hours. The supernatant was collected and centrifuged at 1,000 $\mathrm{g}$ for 10 minutes and then stored at $-80^{\circ} \mathrm{C}$. Phosphorylated epidermal growth factor receptor ( $\mathrm{p}$-EGFR), phosphorylated fibroblast growth factor receptor 2 (p-FGFR2), phosphorylated vascular endothelial growth factor receptor ( $\mathrm{p}$-VEGFR)1, p-VEGFR2, p-MET, phosphorylated human epidermal growth factor receptor 2 (p-HER2), and phosphorylated anaplastic lymphoma kinase (p-ALK) were measured by ELISA according to the manufacturer's protocol.

\section{Xenograft experiment}

Thirty-two BALB/c nude mice, 4-week-old weighing 18-22 g, were purchased from Vital River (Beijing, China). The mice were housed in specific pathogen free conditions and received human care as per the guidelines established by National Science Council, China. HCT-116 cells $\left(2 \times 10^{6}\right)$ were injected subcutaneously into the mice, and tumor volume (TV) was measured weekly and calculated. TV was calculated using the following formula: TV $\left(\mathrm{mm}^{3}\right)=$ $\mathrm{D} \times \mathrm{d}^{2} / 2$, where $\mathrm{D}$ and $\mathrm{d}$ are the longest and shortest diameters, respectively. When the TV reached $200 \mathrm{~mm}^{3}$, the mice were randomized to four groups (six mice/group) and subjected to different treatments (vehicle; 5-Fu: $10 \mathrm{mg} / \mathrm{kg}$ weekly; Andro: $10 \mathrm{mg} / \mathrm{kg}$ daily; 5-Fu plus Andro). The mice were sacrificed and the tumors were removed and measured in 4 weeks. All animal procedures were approved by the Committee on Animal Experimentation of China Medical University.

\section{Terminal dUTP nick end-labeling (TUNEL) assay and immunohistochemistry}

The tumor tissue sections were derived from five randomly selected fields for the TUNEL assay. The assay was used for in situ apoptosis detection via labeling the DNA strand breaks on tissue sections of tumors from control, 5-Fu, Andro, and 5-Fu plus Andro-treated mice according to the manufacturer's instructions (Hoffman-La Roche Ltd, Basel, Switzerland). Briefly, we dewaxed the tumor slides in xylene, hydrated using graded ethanol. The paraffin sections were pretreated with proteinase $\mathrm{K}$ for 30 minutes at $37^{\circ} \mathrm{C}$, then treated with $\mathrm{TdT}$ and incubated in 3\% hydrogen peroxide for 5 minutes. Later, the paraffin sections were treated with peroxidaseconjugated antibody for 10 minutes at room temperature, and incubated with horseradish peroxidase-conjugated secondary antibodies for 2 hours at $37^{\circ} \mathrm{C}$. DAB solution was used to stop the reaction. The results were imaged using an optical microscope (Nikon Eclipse Ti-S).

\section{Statistical analyses}

Results are expressed as the mean \pm SD. Statistical data analysis was performed using GraphPad Prism 5.00 statistical package (GraphPad Software Inc). Student's $t$-test was 
applied to investigate the significant difference among each group. All experiments were performed more than three individual times. $p<0.05$ was regarded as statistically significant.

\section{Results}

\section{Andro synergistically enhanced the antitumor effects of 5-Fu on HCT-II6 cells}

To determine the effect of 5-Fu against HCT-116 cells, the cell viability assay was performed using MTT assay.
The cytotoxic effect of 5-Fu and combination treatments in HCT-116 cells is depicted in Figure 1A and B. The obtained results revealed that the growth of cells was inhibited by $5-\mathrm{Fu}$ in a time-dependent manner. The $\mathrm{IC}_{50}$ values of 5 -Fu were $18.7,8.68$, and $4.28 \mu \mathrm{M}$, corresponding to exposure times of 24, 48, and 72 hours, respectively. Meanwhile, the growth rates exhibited a dose-dependent increase ranging from 0 to $30 \mu \mathrm{M}$ (Figure 1A).

In order to determine the synergistic antitumor effect of Andro on HCT-116 cells, the MTT assay was used and CI was measured. The cells were treated with Andro ranging
A

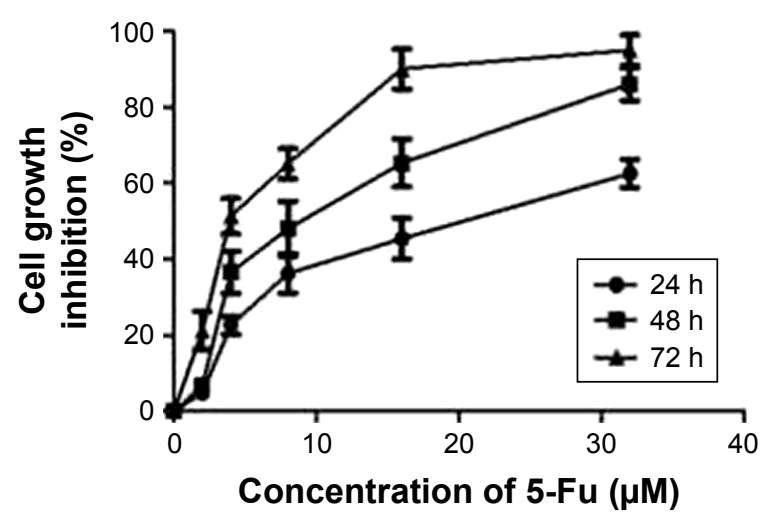

C

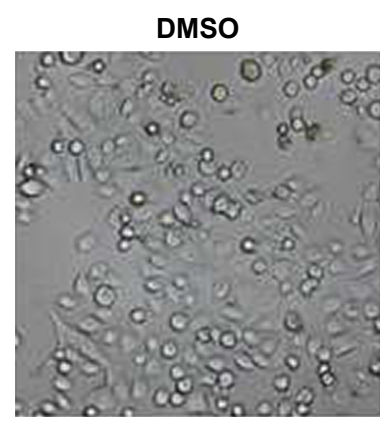

$10 \mu \mathrm{M}$ Andro

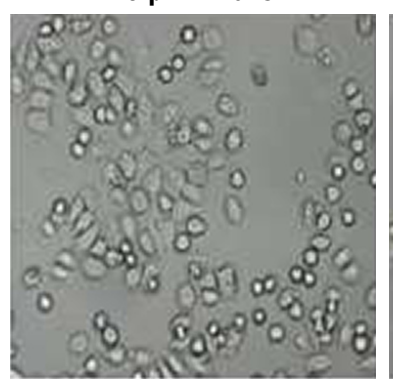

$4.28 \mu \mathrm{M} 5-\mathrm{Fu}$

$5-\mathrm{Fu}+10 \mu \mathrm{M}$ Andro

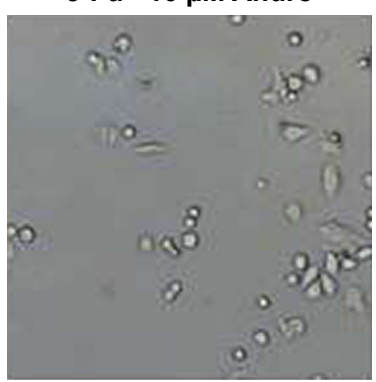

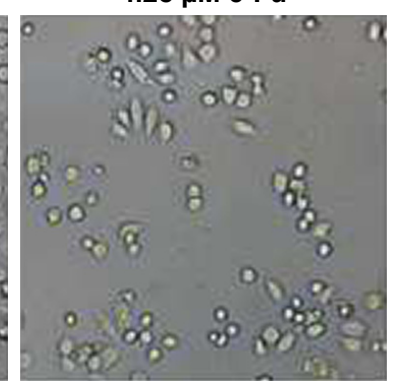
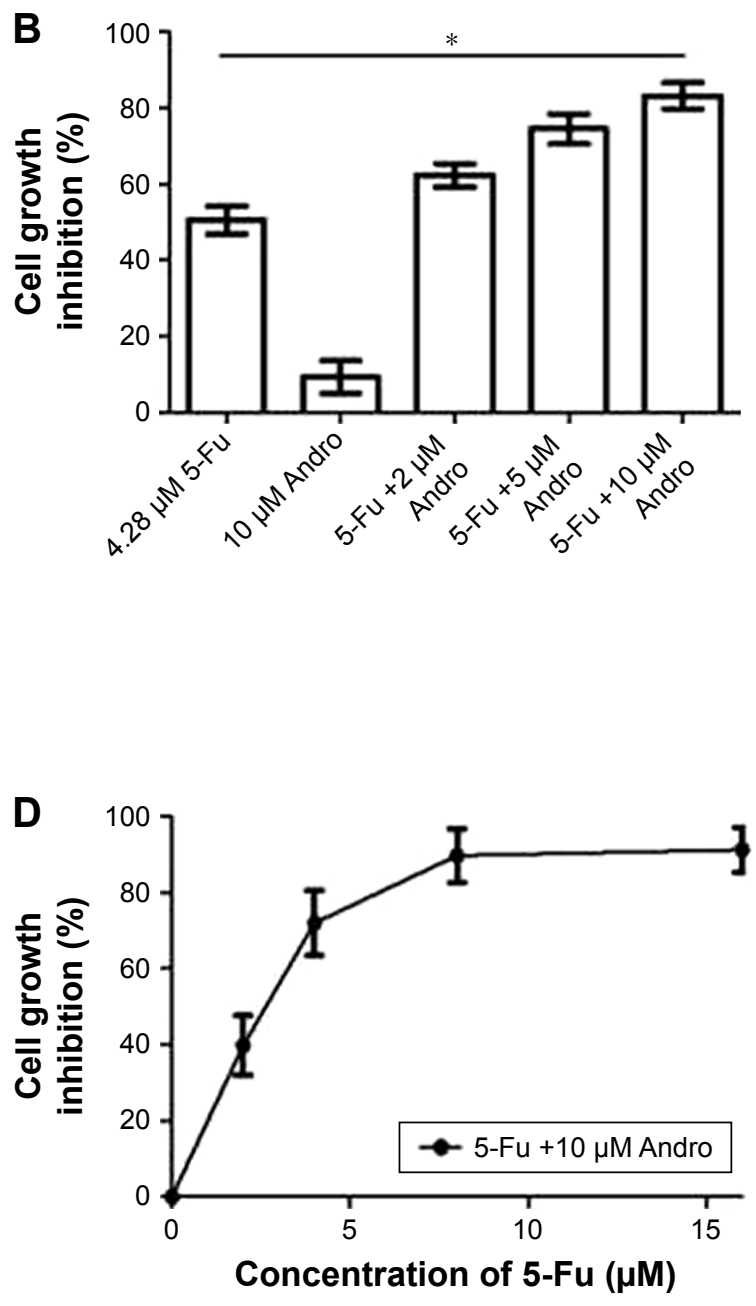

Figure I Andro synergistically enhanced the antitumor effects of 5-Fu on HCT-II 6 cells.

Notes: (A) HCT-I 16 cells were treated with 5 -Fu ranging from 0 to $40 \mu \mathrm{M}$, respectively, for 24,48 , and 72 hours. The cell growth inhibition rate was measured by MTT assay. (B) The cells were treated with 5-Fu, Andro, or 5-Fu plus different concentrations of Andro, respectively, for 24, 48 , and 72 hours. The cell growth inhibition rate was measured by MTT assay. (C) The cells were treated with 5-Fu, Andro, or Andro plus 5-Fu for 72 hours. Morphological changes were observed using a microscope at $200 \times$ magnification. (D) HCT-II6 cells were treated with 5-Fu ranging from 0 to $16 \mu$ M combined with $10 \mu$ M Andro for 72 hours. Each experiment was repeated more than three times independently. (A, B, and D) Data are shown as mean \pm SD, $n=3, *_{p}<0.05$.

Abbreviations: Andro, andrographolide; MTT, 3-(4,5-dimethyl-2-thiazolyl)-2,5-diphenyl-2H-tetrazolium bromide; 5-Fu, 5-fluorouracil; SD, standard deviation; DMSO, dimethyl sulfoxide. 
Table I IC $\mathrm{I}_{50}$ and $\mathrm{Cl}$ values for 5 -Fu single dose (from 0 to $32 \mu \mathrm{M}$ ) or 5 -Fu plus Andro (10 $\mu \mathrm{M})$ in HCT-I I 6 cells in 72 hours

\begin{tabular}{lll}
\hline Dose strategy & IC $_{50}$ values in $72 \mathbf{h}(\mu \mathrm{M})$ & $\mathbf{C l}$ values \\
\hline $5-\mathrm{Fu}$ & 4.28 & - \\
$5-\mathrm{Fu}+10 \mu \mathrm{M}$ Andro & 2.26 & - \\
$4.28 \mu \mathrm{M} 5-\mathrm{Fu}+10 \mu \mathrm{M}$ Andro & & 0.78 \\
\hline
\end{tabular}

Abbreviations: Andro, andrographolide; 5-Fu, 5-fluorouracil; $\mathrm{Cl}$, combination index.

from 2 to $10 \mu \mathrm{M}$ and adding $4.28 \mu \mathrm{M}$ 5-Fu for 72 hours. The results revealed that $10 \mu \mathrm{M}$ Andro exhibited no cytotoxicity against HCT-116 cells, but enhanced cell death induced by 5 -Fu in a dose-dependent manner (Figure 1B). In addition, the $\mathrm{IC}_{50}$ value of the combination treatment was $2.26 \mu \mathrm{M}$, which is lower than that of $5-\mathrm{Fu}$ treated alone (Table 1; Figure 1D).

In order to determine if Andro synergistically enhanced the antitumor effects of 5-Fu in HCT-116 cells, the CI values were calculated. The CI value of the combination of Andro with $5-\mathrm{Fu}$ was 0.78 , which indicated that the combination treatment presented a positive interaction between the two drugs, and confirmed a significantly synergistic antitumor effect against HCT-116 cells in vitro (Table 1). Morphologic changes were observed with 5-Fu, Andro alone, and the combination of 5-Fu with Andro, in comparison to the $0.1 \%$ DMSO which acted as the control group. The results of the morphologic changes were consistent with the cell viability assay (Figure $1 \mathrm{C}$ ). These results indicated that Andro synergistically enhanced the antitumor effect of 5-Fu on HCT-116 cells.

\section{5-Fu-induced apoptosis was enhanced by Andro in HCT-I 16 cells}

We next investigated if apoptosis was involved in the process induced by $5-\mathrm{Fu}$, Andro, or the combination treatment in HCT-116 cells. The results showed that 5-Fu induced HCT-116 cells apoptosis, while the combination treatment of 5-Fu with Andro could significantly increase the apoptosis rate in comparison with 5-Fu treated alone (Figure 2A). In addition, the number of early apoptotic cells induced by the combination treatment was much more than 5-Fu treatment (Figure 2A). To further understand the mechanism of the combination treatment of 5-Fu with Andro in the cells, we evaluated the caspase- 3 activity via Western blot assay. The results showed that the expression of cleaved caspase- 3 in the cells was increased when subjected to the combination treatment of 5-Fu with Andro (Figure 2B). In summary,
A
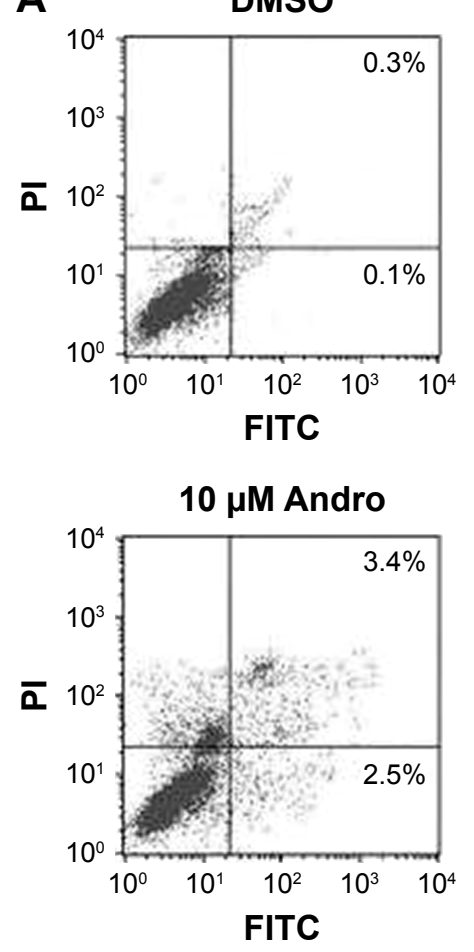
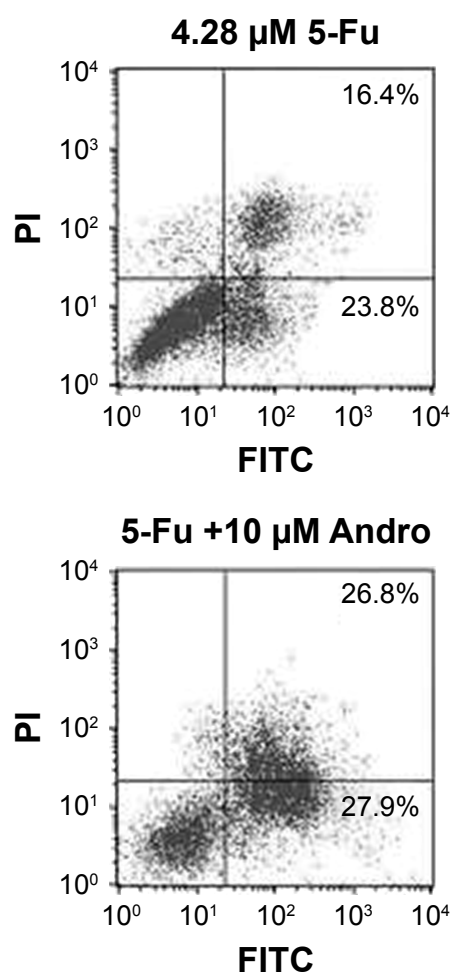

B
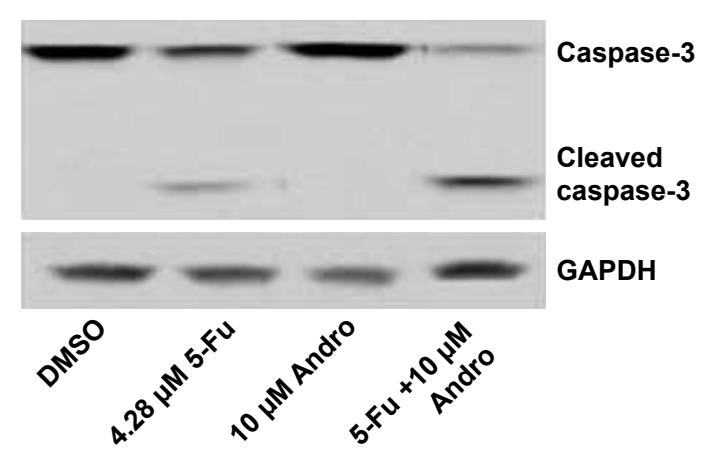

GAPDH

Figure 2 5-Fu-induced apoptotic activities were enhanced by Andro against HCT-II6 cells in vitro.

Notes: (A) HCT-II6 cells were treated with 5-Fu, Andro, or Andro plus 5-Fu for 72 hours. Thereafter, the cells were collected and stained with Annexin V and PI. The apoptotic cells were observed by flow cytometry. (B) The levels of caspase-3 and cleaved caspase-3 were analyzed by Western blot analysis followed by the indicated treatment. Each experiment was repeated more than three times independently.

Abbreviations: Andro, andrographolide; 5-Fu, 5-fluorouracil; SD, standard deviation; PI, propidium iodide; DMSO, dimethyl sulfoxide; FITC, fluorescein isothiocyanate. 
the results indicated that 5-Fu induced HCT-116 cells apoptosis, which was enhanced by Andro significantly via caspase-3 activation.

\section{5-Fu-induced HCT-I I 6 cells apoptosis was enhanced by Andro via inhibition of $\mathrm{p}-\mathrm{MET}$ in vitro}

In order to find out the underlying mechanism of apoptosis induced by the combination of 5-Fu with Andro, we performed the ELISA assay to detect the expression levels of seven types of receptor tyrosine proteins. The results showed that the expression level of p-MET in HCT-116 cells was decreased when subjected to the combination compared with other treatments (Figure 3A). Moreover, the Western blot assay was carried out to further confirm the expression level of p-MET. Results showed that the combination treatment significantly decreased the expression of p-MET in the cells, while the level of c-MET was not changed. In addition, the level of p-AKT and p-ERK was also downregulated by the combination treatment in the cells (Figure 3B). Moreover, cell growth inhibition was reversed by $10 \mathrm{ng}$ HGF (Figure 3C). These data indicated that the synergistic antitumor effect of Andro in HCT-116 was due to p-MET downregulation.

\section{Andro enhanced the antitumor effect of 5 -Fu on HCT-I I 6 xenograft in vivo}

Tumor xenografts transplanted by HCT-116 cells were used to evaluate the antitumor effect of 5-Fu treated alone or the combination of 5-Fu with Andro in vivo. After 4 weeks treatments, the TVs were measured and calculated weekly. The results showed that 5-Fu treatment significantly inhibited tumor growth compared with the control as time progressed. Furthermore, Andro itself demonstrated very limited anti-growth activity on HCT-116 tumor, while the combination treatment showed strong antitumor effects (Figure 4). The results indicated that Andro had a dramatically synergistic antitumor effect with 5-Fu on the HCT-116 xenograft model in vivo.

\section{5-Fu-induced tumor apoptosis was enhanced by Andro due to P-MET level downregulation in vivo}

For determining the mode of cell death in vivo, TUNEL and immunohistochemical assays were performed. We detected the TUNEL positive cells in tumor tissues to evaluate the apoptosis in vivo. The results showed that 5-Fu treated alone increased apoptotic cells in vivo, while the combination treatment of 5-Fu with Andro induced a much higher apoptosis rate (Figure 5A and B). Then we assessed the expression levels of p-MET and c-MET in these tumors and found that the combination treatment of 5-Fu with Andro exerted significant inhibition of p-MET, p-AKT, and p-ERK expression, which was associated with a decreased of TV (Figure 5C). These results were consistent with the in vitro results. Collectively, the results indicated that 5 -Fu-induced tumor apoptosis was enhanced by Andro due to p-MET level downregulation in vivo.

\section{Discussion}

CRC is the fourth cause of cancer-related death worldwide every year. Up to date, 5-Fu is still the most important chemotherapeutic drug to treat CRC. However, the resistance and serious side effects of 5-Fu are the major problems to solve. ${ }^{20}$ Therefore, it is necessary to determine a safe and effective agent to enhance 5-Fu sensitivity. Our study here aimed to investigate the antitumor effects and mechanisms of 5-Fu plus Andro on CRC in vitro and in vivo. Our results showed that low dose of Andro alone had very limited anti-proliferation effect on HCT-116 cells, but could be a potential agent to enhance the antitumor effect of 5-Fu on HCT-116 cells. The results were supported by the activation of cleaved caspase- 3 and an increased apoptosis rate. Furthermore, we detected the underlying mechanisms of epithelial growth factor protein by using ELISA assay, and observed p-MET downregulation by the synergistic treatment of Andro with 5-Fu.

c-MET is a receptor tyrosine-kinase of HGF, has been proven to be involved in drug resistance, and can activate many downstream signaling pathways such as ERK1/2 and PI3K/AKT. ${ }^{21}$ Additionally, it can promote tumor growth via affecting proliferation, anti-apoptosis, invasion, and angiogenesis. ${ }^{22}$ To determine the target underlying mechanism for 5-Fu plus Andro in advanced HCT-116 cells, we first conduct an ELISA assay to examine the expression of epidermal growth factors p-EGFR, p-FGFR2, p-VEGFR1, p-MET, p-HER2, p-ALK, and p-VEGFR2 in HCT-116 cells. Our results highlighted p-MET as an important target in HCT-116 cells; especially, the level of p-MET was significantly downregulated in HCT-116 cells when treated with 5-Fu plus Andro. In addition, our results showed that Andro downregulated the level of p-MET compared with the control group, while the expression level of p-MET was further downregulated by 5-Fu plus Andro. The results indicated that p-MET was involved in the synergistic apoptotic activity in HCT-116 cells. 


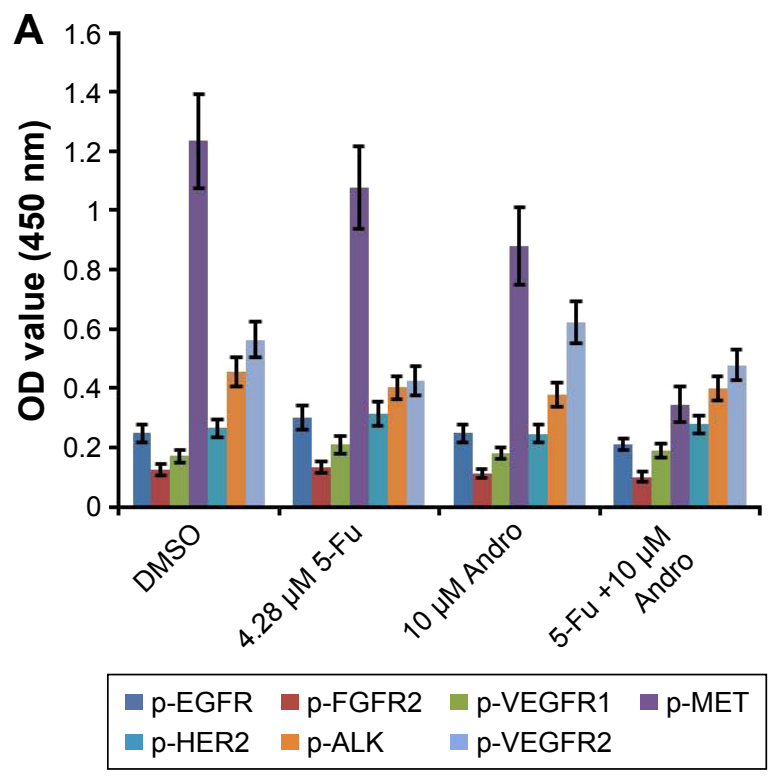

B

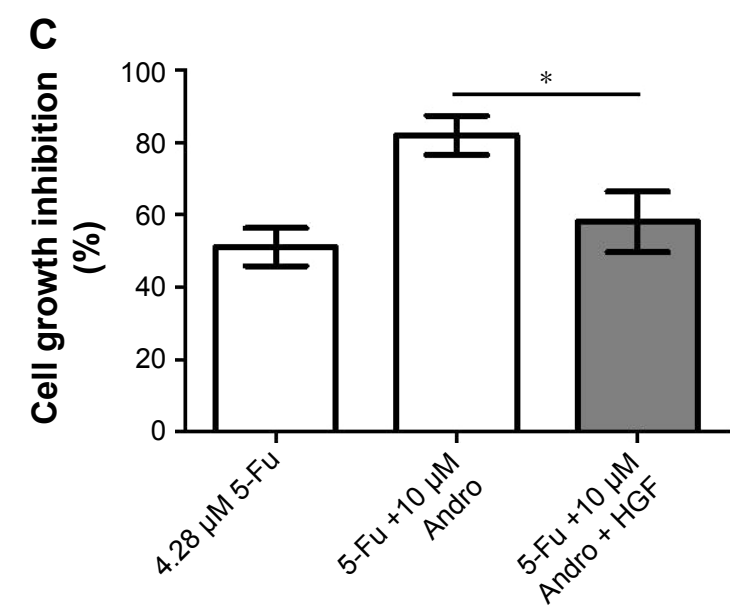

Figure 3 5-Fu-induced HCT-II6 cells apoptosis was enhanced by Andro by inhibition of c-MET pathway in vitro.

Notes: (A) HCT-II 6 cells were treated with 5-Fu, Andro, or Andro plus 5-Fu for 72 hours. The expression levels of epidermal growth factors P-EGFR, p-FGFR2, P-VEGFRI, p-MET, p-HER2, p-ALK, and p-VEGFR2 were measured using ELISA kit. (B) The expression levels of c-MET, p-MET, p-AKT, and p-ERK were analyzed by Western blot analysis. (C) The HCT-II6 cells were treated with 5-Fu, Andro plus 5-Fu, or three combinations for 72 hours. The cell growth inhibition rate was measured by MTT assay. Each experiment was repeated more than three times independently. ( $A$ and $\mathbf{C}$ ) Data are shown as mean $\pm \mathrm{SD}, \mathrm{n}=3,{ }^{*} \mathrm{p}<0.05$.

Abbreviations: Andro, andrographolide; MTT, 3-(4,5-dimethyl-2-thiazolyl)-2,5-diphenyl-2H-tetrazolium bromide; 5-Fu, 5-fluorouracil; SD, standard deviation; DMSO, dimethyl sulfoxide; p-EGFR, phosphorylated epidermal growth factor receptor; p-FGFR2, phosphorylated fibroblast growth factor receptor 2; p-VEGFRI, phosphorylated vascular endothelial growth factor receptor I; P-MET, phosphorylated cellular-mesenchymal to epithelial transition factor; p-HER2, phosphorylated human epidermal growth factor receptor 2; p-ALK, phosphorylated anaplastic lymphoma kinase; p-VEGFR2, phosphorylated vascular endothelial growth factor receptor 2; ELISA, enzyme linked immunosorbent assay; OD, optical density; HGF, hepatocyte growth factor.

A previous study has reported that 5-Fu plus Andro cotreatment determined the apoptosis level of HCT-116 cells with increased level of Bax. ${ }^{14}$ c-MET activation induces diverse signaling cascades leading to cell proliferation, invasion, metastasis, angiogenesis, and survival. ${ }^{23,24}$ Meanwhile, it has been reported that c-MET can activate downstream signaling pathways such as ERK $1 / 2$ and PI3K/AKT. In the current study, both $\mathrm{p}$-ERK $1 / 2$ and $\mathrm{p}$-AKT were significantly inhibited by the combination treatment in vitro and in vivo. These results confirmed that the synergistic antitumor effect of Andro had a close relationship with c-MET pathway downregulation. However, detailed mechanisms of why 5-Fu plus Andro co-treatment dramatically inhibited the c-MET pathway are unclear and need more experiments for clarification.

In summary, we demonstrated that Andro synergistically enhanced the apoptotic rate of 5-Fu on HCT-116 cells, via downregulating the c-MET pathway in vivo and in vitro. Our results provide evidence for the possible clinical application of Andro for enhancing the antitumor effect of 5-Fu on CRC treatment. 


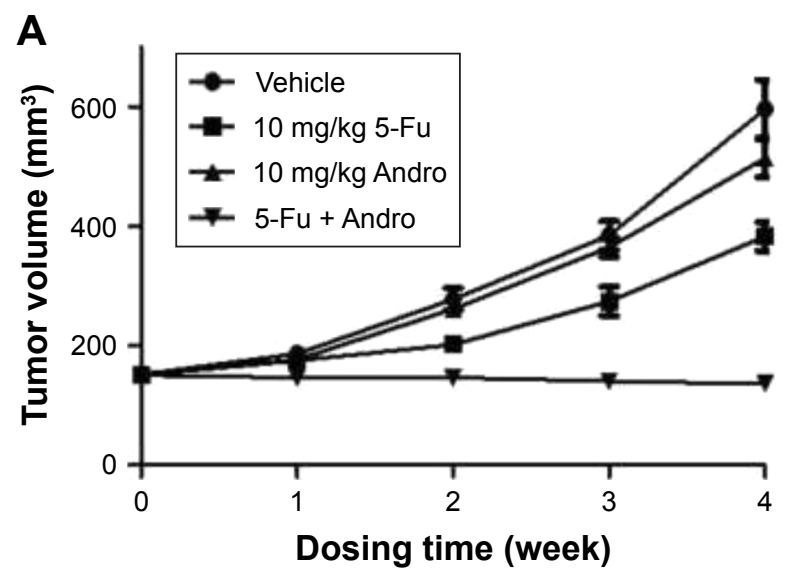

B

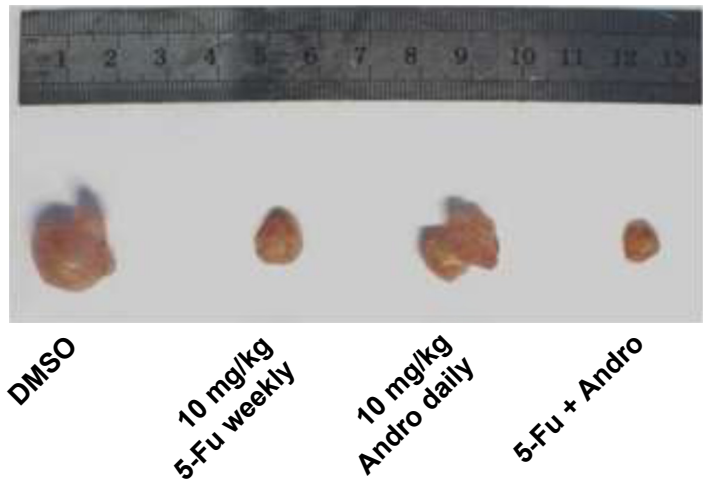

Figure 4 Andro enhanced the antitumor effect of 5-Fu on HCT-II6 xenograft in vivo.

Notes: (A) HCT-II 6 tumor bearing mice were administered vehicle, $10 \mathrm{mg} / \mathrm{kg} 5$-Fu (weekly, ip), $10 \mathrm{mg} / \mathrm{kg}$ Andro (daily, po), or the combination treatment of 5-Fu with Andro. Tumor volumes were monitored weekly. Data are shown as mean $\pm \mathrm{SD}, \mathrm{n}=6$. (B) The tumors were collected after 4 weeks treatment and weighted. Abbreviations: Andro, andrographolide; 5-Fu, 5-fluorouracil; SD, standard deviation; DMSO, dimethyl sulfoxide; ip, intraperitoneal; po, per oral.

A

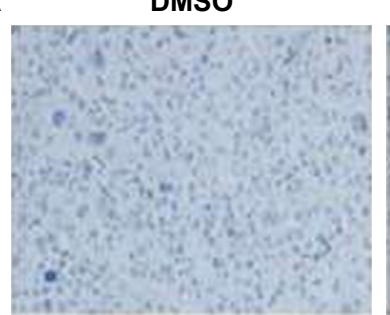

$10 \mu \mathrm{M}$ Andro

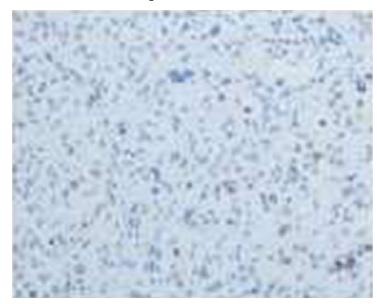

$4.28 \mu \mathrm{M} 5-\mathrm{Fu}$

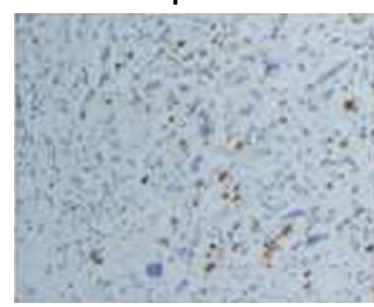

5-Fu +10 $\mu \mathrm{M}$ Andro

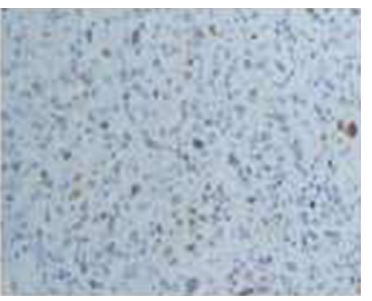

B

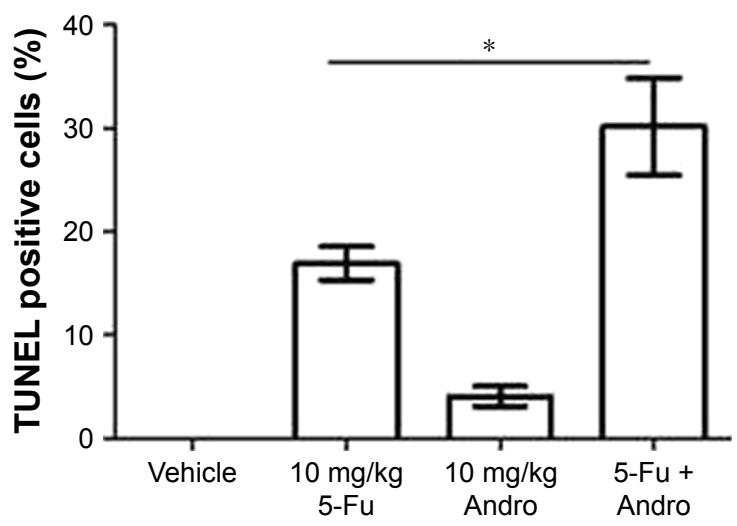

C

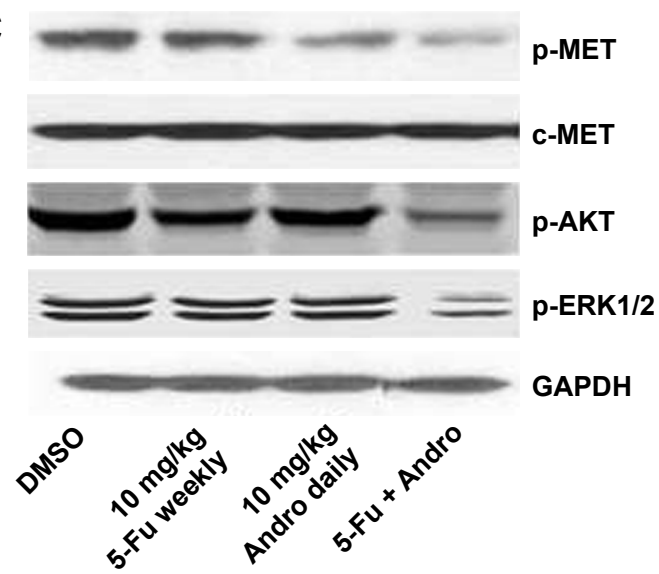

Figure 5 5-Fu-induced tumor apoptosis was enhanced by Andro due to p-MET level downregulation in vivo.

Notes: (A, B) The mice were sacrificed after 4 weeks of treatment; the tumors were removed and dewaxed. The apoptotic rate was measured using TUNEL assay and immunohistochemistry. (B) Data are shown as mean $\pm \mathrm{SD}, \mathrm{n}=3,{ }^{*} \mathrm{p}<0.05$. (C) The expression levels of c-MET, p-MET, $\mathrm{p}-\mathrm{AKT}$, and $\mathrm{p}$-ERK were analyzed by Western blot in tumor tissue. Each experiment was repeated more than three times independently.

Abbreviations: Andro, andrographolide; 5-Fu, 5-fluorouracil; SD, standard deviation; DMSO, dimethyl sulfoxide; TUNEL, terminal dUTP nick end-labeling; p-MET, phosphorylated cellular-mesenchymal to epithelial transition factor. 


\section{Disclosure}

The authors report no conflicts of interest in this work.

\section{References}

1. PDQ Screening and Prevention Editorial Board. Colorectal cancer screening $\left(\mathrm{PDQ}^{\circledR}\right)$ : health professional version. In: $P D Q$ Cancer Information Summaries. Bethesda, MD: National Cancer Institute; 2002.

2. Jemal A, Bray F, Center MM, Ferlay J, Ward E, Forman D. Global cancer statistics. CA Cancer J Clin. 2011;61:69-90.

3. Yatsuoka T, Nishimura Y, Sakamoto H, Tanaka Y, Kurozumi M. [Lymph node metastasis of colorectal cancer with submucosal invasion]. Gan To Kagaku Ryoho. 2013;40:2041-2043. Japanese.

4. Shi Y, Huang XX, Chen GB, et al. Dragon (RGMb) induces oxaliplatin resistance in colon cancer cells. Oncotarget. 2016;7:48027-48037.

5. Wiegering A, Matthes N, Muhling B, et al. Reactivating p53 and inducing tumor apoptosis (RITA) enhances the response of RITA-sensitive colorectal cancer cells to chemotherapeutic agents 5-fluorouracil and oxaliplatin. Neoplasia. 2017;19:301-309.

6. Onozawa H, Saito M, Saito K, et al. Annexin A1 is involved in resistance to 5-Fu in colon cancer cells. Oncol Rep. 2017;37:235-240.

7. Negi AS, Kumar JK, Luqman S, Shanker K, Gupta MM, Khanuja SP. Recent advances in plant hepatoprotectives: a chemical and biological profile of some important leads. Med Res Rev. 2008;28: 746-772.

8. Malahubban M, Alimon AR, Sazili AQ, Fakurazi S, Zakry FA. Phytochemical analysis of Andrographis paniculata and Orthosiphon stamineus leaf extracts for their antibacterial and antioxidant potential. Trop Biomed. 2013;30:467-480.

9. Wen L, Xia N, Chen X, et al. Activity of antibacterial, antiviral, antiinflammatory in compounds andrographolide salt. Eur J Pharmacol. 2014;740:421-427.

10. Khan A, Sharma P, Khan F, Ajayakumar PV, Shanker K, Samad A. In silico and in vitro studies on Begomovirus induced andrographolide biosynthesis pathway in Andrographis paniculata for combating inflammation and cancer. Mol Inform. 2016;35:253-261.

11. Zhang M, Xue E, Shao W. Andrographolide promotes vincristineinduced SK-NEP-1 tumor cell death via PI3K-AKT-p53 signaling pathway. Drug Des Devel Ther. 2016;10:3143-3152.
12. Mi S, Xiang G, Yuwen D, et al. Inhibition of autophagy by andrographolide resensitizes cisplatin-resistant non-small cell lung carcinoma cells via activation of the Akt/mTOR pathway. Toxicol Appl Pharmacol. 2016;310:78-86.

13. Banerjee A, Ahmed H, Yang P, Czinn SJ, Blanchard TG. Endoplasmic reticulum stress and IRE-1 signaling cause apoptosis in colon cancer cells in response to andrographolide treatment. Oncotarget. 2016;7: 41432-41444.

14. Wang W, Guo W, Li L, et al. Andrographolide reversed 5-Fu resistance in human colorectal cancer by elevating BAX expression. Biochem Pharmacol. 2016;121:8-17.

15. Chou TC, Talalay P. Quantitative analysis of dose-effect relationships: the combined effects of multiple drugs or enzyme inhibitors. Adv Enzyme Regul. 1984;22:27-55.

16. Chou TC. Drug combination studies and their synergy quantification using the Chou-Talalay method. Cancer Res. 2010;70:440-446.

17. Chou TC. Preclinical versus clinical drug combination studies. Leuk Lymphoma. 2008;49:2059-2080.

18. Cummings BS, Schnellmann RG. Measurement of cell death in mammalian cells. Curr Protoc Pharmacol. 2004;12:12-18.

19. Nguyen VP, Kim SW, Kim H, et al. Biocompatible astaxanthin as a novel marine-oriented agent for dual chemo-photothermal therapy. PLoS One. 2017;12(4):e174687.

20. Temraz S, Mukherji D, Alameddine R, Shamseddine A. Methods of overcoming treatment resistance in colorectal cancer. Crit Rev Oncol Hematol. 2014;89:217-230.

21. Engelman JA, Zejnullahu K, Mitsudomi T, et al. MET amplification leads to gefitinib resistance in lung cancer by activating ERBB3 signaling. Science. 2007;316:1039-1043.

22. Birchmeier C, Birchmeier W, Gherardi E, Vande Woude GF. Met, metastasis, motility and more. Nat Rev Mol Cell Biol. 2003;4:915-925.

23. Pomerleau V, Landry M, Bernier J, Vachon PH, Saucier C. Met receptor-induced Grb2 or Shc signals both promote transformation of intestinal epithelial cells, albeit they are required for distinct oncogenic functions. BMC Cancer. 2014;14:240.

24. Bernier J, Chababi W, Pomerleau V, Saucier C. Oncogenic engagement of the Met receptor is sufficient to evoke angiogenic, tumorigenic, and metastatic activities in rat intestinal epithelial cells. Am J Physiol Gastrointest Liver Physiol. 2010;299:G677-G686.

\section{Publish your work in this journal}

Drug Design, Development and Therapy is an international, peerreviewed open-access journal that spans the spectrum of drug design and development through to clinical applications. Clinical outcomes, patient safety, and programs for the development and effective, safe, and sustained use of medicines are the features of the journal, which has also been accepted for indexing on PubMed Central. The manuscript management system is completely online and includes a very quick and fair peer-review system, which is all easy to use. Visit http://www.dovepress.com/testimonials.php to read real quotes from published authors. 